\title{
Chemoresistive sensors for colorectal cancer preventive screening through fecal odor: double-blind approach
}

\author{
G. Zonta ${ }^{1,3}$, G. Anania ${ }^{1}$, M. Astolfi', C. Feo ${ }^{1,5}$, S. Gherardi ${ }^{3}$, A. Giberti3,4, V. Guidi' ${ }^{1}$, N. Landini, ${ }^{1,3}$, C. \\ Palmonari1,2, A. de Togni² and C. Malagù ${ }^{1,3}$ \\ ${ }^{1}$ University of Ferrara, Via Savonarola 9 - 44121 Ferrara, Italy \\ ${ }^{2}$ Department of Public Health (AUSL) - UO Igiene Pubblica - Via Fausto Beretta, 7 - 44121 Ferrara, Italy \\ ${ }^{3}$ SCENT S.r.I., Via Quadrifoglio 11 - 44124 Ferrara, Italy \\ ${ }^{4}$ MIST E-R s.c.r.I., Via P. Gobetti 101, 40129 Bologna, Italy \\ ${ }^{6}$ Ospedale del Delta, Via Valle Oppio, 2 - Lagosanto FE, Italy \\ giulia.zonta@unife.it
}

\begin{abstract}
:
Preventive screening of tumors is fundamental to prevent their degeneration. The curable rate of colorectal cancer (CRC), identified at stage I is up to $90 \%$. Here the results obtained so far in the clinical validation Protocol, using a device hosting an array of chemoresistive semiconductor gas sensors, are shown. The Protocol has been started in May 2016. The device can identify the difference between the odor of feces from two categories of people: healthy subjects, and subjects affected by high-risk adenomas or tumors. Moreover, we are calibrating the instrument so that it can also recognize low-risk adenomas. The tests are compared to fecal occult blood test and colonoscopy (gold-standard) results. The tests are still ongoing since 31 December 2018.
\end{abstract}

Key words: Sensors, biomarkers, colorectal cancer, feces, device.

\section{Introduction}

The odor of fecal samples can be modified by the presence of colorectal-cancer (CRC) in human body. This alteration is due to two main processes: i) the peroxidation of cell membranes; ii) the alteration of cellular metabolism. SCENT A1 [1] is a patented device (both in Italy and Europe), realized at the Sensors Laboratory (SL) of the University of Ferrara, capable of performing a preventive screening of CRC. It is composed by a pneumatic system that conveys fecal exhalations to the sensing core, consisting of an array of five chemoresistive nanostructured sensors, made of semiconductor materials. A specifically realized data acquisition software registers sensor response curves, which are then analyzed by a specific algorithm. Work is in progress to make the process user friendly.

\section{Experimental section}

The chosen materials are of metal oxide type, with average grain dimensions between 30-63 $\mathrm{nm}$. The materials are: iron and samarium oxide, two different solid solutions of tin and titanium oxides, the second with the addition of gold nanoparticles, and indium oxide. Sensors have been chosen after a feasibility study in collaboration with the Department of Morphology, Surgery and Experimental Medicine of the University of Ferrara (UNIFE), described in detail in reference [2]. The data analysis method tested, that lead to the preliminary results of the first part of the protocol, were principal component analysis (PCA) and quadratic discriminant analysis (QDA), performed on responses. The response $(R)$ is calculated, for $n$-type sensors, as $\Delta G / G$, where $\Delta G$ is the difference between conductance with VOCs-contaminated air $\left(G_{g a s}\right)$ and the conductance in environmental air $(G)$. For $p$-types, $R$ is the negative reciprocal. In May 2016, the clinical validation Protocol, that involves S. Anna Hospital of Ferrara, UNIFE, Ospedale del Delta of Lagosanto and Dipartimento Sanità Pubblica (AUSL) of Ferrara and the startup SCENT S.r.I, started. SCENT A1 experimental test is proposed to all screening users in Ferrara (49-69 years old), who resulted positive to fecal occult blood test (FOBT), before undergoing colonoscopy (gold standard). A specific software, realized by us in LabView, allows to identify four parameters related to the response curve of each sensor (response at 90\%, integral, derivative and time). The total of 20 parameter is then analysed with support vector machine (SVM) method [4]. 
The logic behind the Protocol consists of two steps: a) creation of a statistical database and b) double-blind check. In the first part of the analysis, SVM has been employed as an indicator of how well data is classified by labelling populations into three categories, as indicated by colonoscopy outputs: i) cancer and high-risk adenomas (POS); ii) low-risk adenomas (LOW); iii) healthy subjects (NEG). A total of 275 tests with SCENT A1 has been performed, from which 91 comparisons with colonoscopy have already been made. The statistic is progressively updated even if the process is not fast, due to the technical times to obtain the results of the gold-standard. However, we expect within the year to get the numbers needed for clinical validation. To date, the method correctly classified the $100 \%$ of $\mathrm{HS}$, the $100 \%$ of CRC+HR and the $60 \%$ of LR (confusion matrix reported in Figure 1). This first approach is fundamental to set-up the database that will serve for double-blind tests. About this, by employing the previous statistics [4] without the inclusion of the last three additional positive samples, we have made a double-blind test. The three positives, two infiltrating adenocarcinomas (samples 1,2) and an advanced adenoma (sample 3) have been tested with the algorithm without any label inserted a-priori. The first two have been correctly classified as positives, while the third resulted in a false negative (probability of being positive of $43 \%$ ). However, despite the error, after having observed the classification probabilities related to each sample (see Table 1) and the risk level of each cancer (other information not reported for privacy reasons), what emerges is that sample 3 has a riskdegree smaller than the other two, in fact it is an advanced adenoma.

In the analysis, we have grouped the samples into three classes, because of the low number of positives with respect to negatives. Our next goal is to increase, moth by month, the statistics on positives to increment the level of detail in the classification. This will serve to make the system able of defining more CRCclasses, divided by risk, in line with current medical definitions and not only a single threshold value.

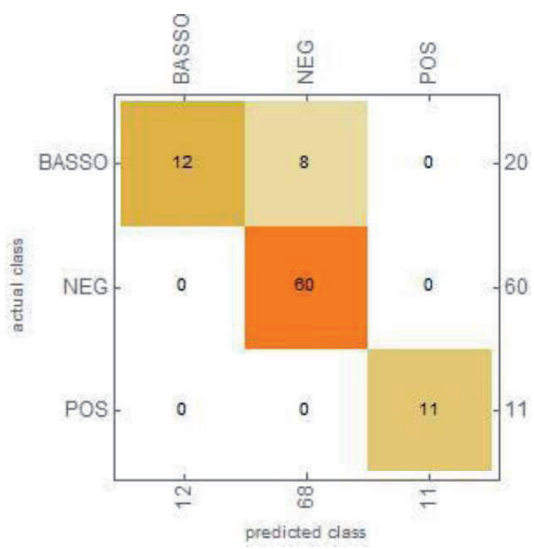

Figure 1: confusion matrix made with 91 samples tested with SCENT A1, already resulted positives to FOBT and then compared with the gold-standard (colonoscopy).

\begin{tabular}{|c|c|c|c|c|}
\hline SAMPLE & $\begin{array}{c}\text { CANCER } \\
\text { TYPE }\end{array}$ & $\begin{array}{c}\text { LOW } \\
\%\end{array}$ & $\begin{array}{c}\text { NEG } \\
\%\end{array}$ & POS\% \\
\hline 1 & $\begin{array}{c}\text { infiltrating } \\
\text { adenocarcinoma }\end{array}$ & 32 & 27 & 41 \\
\hline 2 & $\begin{array}{c}\text { infiltrating } \\
\text { adenocarcinoma }\end{array}$ & 6 & 15 & 79 \\
\hline 3 & $\begin{array}{c}\text { advanced } \\
\text { adenoma }\end{array}$ & 31 & 43 & 26 \\
\hline
\end{tabular}

Table 1: pathology and classification probabilities associated to the three POS samples, resulted with the SCENT A1 algorithm in double-blind. Samples 1,2 are correctly classified as positives, taking on account the database collected before during the Protocol.

\section{Conclusions}

Here some new results of the clinical validation protocol of the device SCENT A1 for the preventive screening of $C R C$ are proposed. The protocol will be active since the end of 2018 , to obtain the statistics for validating the device. Results are promising and show the capability of this test of reducing the number of false positives of FOBT of about $75 \%$, if employed together. This will be useful to avoid unnecessary colonoscopy on healthy subjects. A wider statistic will be obtained during the next months.

\section{References}

[1] SCENT A1, patent number: RM20144000595, patent and intellectual property of SCENT S.r.I., European internationalization;

[2] G. Zonta, G. Anania, B. Fabbri, A. Gaiardo, S. Gherardi, A. Giberti, N. Landini, C. Malagù, L. Scagliarini, V. Guidi, Preventive screening of colorectal cancer with a device based on chemoresistive sensors Sensors and Actuators B, 238, 1098-110, 2016;

[3] N. Landini, G. Zonta, C. Malagù (2015), Detection of tumor markers on feces with nanostructured sensors, Scholars' Press, ISBN-13: 978-3-639-76538-0;

[4] G. Zonta, G. Anania, C. Feo, A. Gaiardo, S. Gherardi, A. Giberti, V. Guidi, N. Landini, C. Palmonari, L. Ricci, A. de Togni, C. Malagù, Use of gas sensors and FOBT for the early detection of colorectal cancer, accepted by Sensors and Actuators B (2018). 